\title{
In-Vitro Magnetoresistive Biosensors for Single Molecular Based Disease Diagnostics: Optimization of Sensor Geometry and Structure
}

\author{
Seongtae Bae \\ Department of Electrical and Computer Engineering, Biomagnetics Laboratory (BML), \\ National University of Singapore, 117576, Singapore \\ Singapore
}

\section{Introduction}

Detection of biological information using magnetoresistance (MR) sensors based on multilayered giant MR (GMR), or exchange biased GMR spin valves, operated by the magnetic field produced by magnetic nanoparticle sensor agents has been paid considerable attention in biomedical sensor technologies (Baselt et al., 1998; Tondra et al., 2000; Rife et al., 2003; Graham et al., 2004). The main reason for this interest is that MR based biosensors provide technical advantages such as high sensitivity, relatively fast and low-volume assay, and easy manipulation of the magnetic sensor agents under the externally applied magnetic field gradients (Graham et al., 2002; Lagae et al., 2002). These advantages lead to improved sensing performance, stimulating the development of functional in-vitro GMR based biosensors to obtain biological information and diagnose diseases more accurately in healthcare (Megen \& Prins, 2005).

However, all the developed GMR biosensors so far were mostly focused on counting or indentifying multiple biomolecules such as a DNA counter and a bead array counter rather than single molecular detection (SMD) (Baselt et al., 1998; Miller et al., 2001; Graham et al., 2003; Li \& Wang, 2003; Schepper et al., 2004; Wang et al., 2005; Shen et al., 2005). The main physical reason for this technical limitation is that the magnetic susceptibility of a single superparamagnetic nanoparticle sensor agent (SPNSA), which can be easily manipulated on the sensor surface without any serious agglomeration and easily retrieve magnetic stray field, is too small to generate a large enough field for acheiving a reasonably high SNR (Singal-to-Noise Ratio). Although superparamagentic microbeads have been attempted to obtain a larger stray field for higher SNR for SMD (Graham et al., 2002; Wirix-Speetiens et al., 2006), these agents were also revealed to have a technical drawback that they can not maintain one to one ratio between the microbead and the biomolecules due to a big mismatch in size. Hence, to develop more powerful in-vitro GMR bisoensor system for SMD, ferrimagentic nanoparticle (FN) sensor agents, which have a high remnant magentization expecting to producing a sufficient stray field for a higher SNR, and a high chemical stability as well as a high biocompatibility with living cells or biological entities, are currently considered as a feasible sensor agent to label the biomolecules. However, the applications of FN to a GMR biosensor agent has been limited for the past few years by a

Source: Intelligent and Biosensors, Book edited by: Vernon S. Somerset,

ISBN 978-953-7619-58-9, pp. 386, January 2010, INTECH, Croatia, downloaded from SCIYO.COM 
techical challenge relevant to a possible particle agglomeration while they are introducing into the microchannel. Fortunetely, as a new functional microfluidic channel with micromagnet, which can allow for effectively manipulating the FNs to flow into the channel one by one, has been recently developed (Lagae et al., 2002; Ramaden et al., 2006; Latham et al., 2007), the interests to apply a single FN sensor agent to an in-vitro GMR biosensor for SMD are dramtically increased in a molecular diagnostic biosensor system.

In this chapter, the physical characteristics of an in-vitro GMR biosensor with an immoblized single FNSA is mainly discussed to provide crucial information how to optimize its strucure for SMD. In chapter 2, the sensing performance of an in-vitro GMR biosensor with an immobilized FNSA or SPNSA is numerically analyzed based on the "Stoner-Wolfarth model" to evaluate which GMR biosensor system is more suitable for SMD. In chapter 3, the optimization of sensor geometry considering the spatial magentic field interaction between the FNSA and the FL as well as the physical correlation between the "effective sesning area" and SNR is introduced to successfully design an in-vitro GMR biosensor enabling to show maximized SNR for SMD. Finally, chapter 4 discusses the newly desgined sensoror structure of an in-vitro GMR biosensor with a specially designed magnetic shield layer (MSL) to explore its technical effectiveness for the diagnostic biosensor applications based on SMD.

\section{An in-vitro GMR biosensor with an immobilized single FNSA or SPNSA for SMD}

To confirm which sensor system is more promising for SMD, the sensing performance of an in-vitro GMR biosensor with an immobilized FNSA or SPNSA should be first clarified. Four physical sensing parameters: $(1)$ the relative MR change $(\delta R)$ relevant to the magnetization angle difference between the free layer (FL) (sensing layer) and the pinned layer in GMR spin-valves, (2) the interaction factor (IF) describing the magnetic spatial interaction between the FL and the nanoparticle sensor agent, (3) the distance between the single immobilized FNSA or SPNSA and the FL, and (4) the practically allowable sensor size considering the physical limit of current sensor fabrication technology, are mainly considered for the comparison of sensing performance. The numerical analysis is based on the "Stoner-Wolfarth model" describing that the magentizations of the FL in the GMR biosensors are coherently rotated under a globally applied magentic field with twodimensional field components, which are homogeneous across the entire FL.

\subsection{Physical model of spatial field distribution on an in-vitro GMR biosensor}

The sensing mechanism of a GMR biosensor with an immobilized single FNSA or SPNA is illustrated in Fig. 1-(a). The sensor agents are captured on the FL surface of GMR biosensor through a bio-recognition process and the resistance of the GMR sensor is accordingly changed by the stray field produced from the magnetic nanoparticles. As shown in Fig. 1, the critical geometry parameters considered for the numerical calculation include the spacing between the SA and the sensor surface (h), and the geometry of FL, e.g. length (L), width (W), and thickness (t). Figure 1-(b) describes the magnetization configuration of FL in the GMR biosensor and the magnetic spatial interaction between the SA and the FL. The magnetization of pinned layer (PL) is exchange biased to the negative transverse direction (-y) and the easy axis (EA) of the FL is along the longitudinal direction $(+x)$ due to shape anisotropy and crystalline anisotropy. To minimize the geometrically-induced magnetostatic coupling 
between the FL and the orthogonally coupled PL, the PL is considered as the "synthetic antiferromagnetic (AFM) layer", e.g. FM/non-magnetic/FM structure. The magnetization of FNSA is aligned in the positive transverse direction (+y) and the SPNSA is magnetized toward the positive transverse direction $(+y)$ by the excitation field $(+y)$ for comparison with the FNSA. In order to establish a physical model for the stray field induced by the magnetic nanoparticles, the single magnetic nanoparticle with spherical shape is assumed to be uniformly magnetized and has an effective dipole magnetic moment $(\mathrm{m})$. The field intensity, $\mathrm{H}(\mathrm{H} \cong \mathrm{B}$ in cgs unit) at a distance $\mathrm{r}$ can be expressed by equation (1) (Besse et al., 2002).

$$
\overrightarrow{H(B)}=\frac{3 \hat{r}(\hat{r} \times m)-m}{r}
$$

The spatial distributions of the stray field induced by the magnetic nanoparticle on the FL of GMR biosensor are numerically calculated by considering the both longitudinal and transverse magnetic field components of an ideal dipole, as illustrated in Fig. 1-(b).

$$
\begin{aligned}
& H_{x}\left(B_{x}\right)=m \cdot \frac{2 x^{2}-y^{2}-z^{2}}{\left(x^{2}+y^{2}+z^{2}\right)^{5 / 2}} \\
& H_{y}\left(B_{y}\right)=m \cdot \frac{3 x y}{\left(x^{2}+y^{2}+z^{2}\right)^{5 / 2}} \\
& H_{z}\left(B_{z}\right)=m \cdot \frac{3 x z}{\left(x^{2}+y^{2}+z^{2}\right)^{5 / 2}}
\end{aligned}
$$

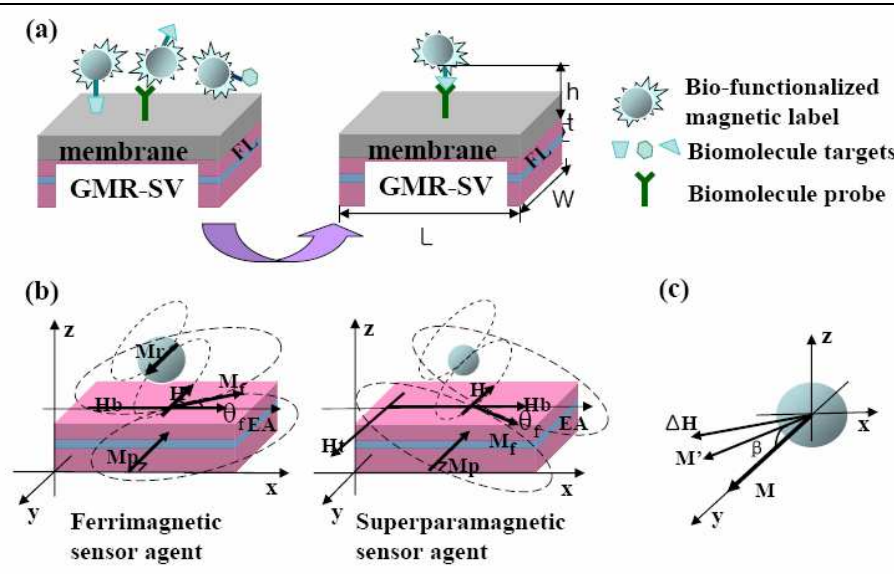

Fig. 1. A schematic diagram of an in-vitro GMR biosensor with a single nanoparticle sensor agent for SMD (a) the molecular recognition process on the GMR biosensor surface, (b) the magnetic spatial interaction between the magnetic nanoparticle agents and the FL of GMR biosensor. All the field components generated from the bias field, $\mathrm{H}_{\mathrm{b}}$, the excitation field, $\mathrm{H}_{\mathrm{t}}$ and the magnetic nanoparticles are illustrated on the FL sensor surface, and (c) a schematic diagram of magnetization configuration of a single magnetic nanoparticle and its variation due to the magnetic dipole interaction caused by the x-component of FL magnetization to the FNSA or the SPNSA. 
The total free energy in the FL is described by equation (3) based on the "Stoner- Wolfarth model" (Stoner \& Wolfarth, 1948).

$$
E=K_{u} \sin ^{2} \theta+\frac{1}{2}\left(N_{z}-N_{x}\right) M_{s} \sin ^{2} \theta-H M_{s} \cos (\psi-\theta)-H_{t} M_{s} \sin \theta-H_{b} M_{s} \cos \theta
$$

Where $H$ is the stray field generated by the magnetic nanoparticles, which can be divided into $H_{x}$ and $H_{y}$, respectively. The demagnetizing factors can be determined by equation (4) (William, 2001).

$$
N_{z}=8 t w / l \sqrt{l^{2}+w^{2}}, N_{z}=8 t w / w \sqrt{l^{2}+w^{2}}
$$

According to the "Stoner- Wolfarth model", the magnetization direction of the FL (FL is in a single domain state as the sensor size is in submicron range in this model) is determined by the minimization of the total free energy as shown in equation (3). Hence, the total free energy with respect to $\theta$ (Fig. 1-(c)) is minimized. Our method is to set up a discrete array of $\theta$. The range of this array is from 0 to $2 п$ and the step size is 0.00001 . With such small step size, the range of $\theta$ can be considered as a continuous range. By substituting the array into equation (3), the minimized energy and the corresponding $\theta$ can be obtained using a simple algorithm. The next step is to use the following equation (5) to calculate the magnetoresistance (MR) ratio based on the magnetization (spin) configuration of GMR biosensor where the magnetization direction of PL is exchange biased to a fixed direction $(-y)$.

$$
\frac{\Delta R}{R}=\left(\frac{\Delta R}{R}\right)_{0} \frac{1-\cos \left(\theta_{f}-\theta_{P}\right)}{2}
$$

Where $\theta_{f}$ and $\theta_{p}$ are the angles of the $\mathrm{FL}$, and the PL with respect to EA, respectively; $(\Delta R / R)_{0}$ is the MR ratio of the GMR biosensor when the FL and PL is antiparallel with each other, which is also the maximum MR ratio for a GMR biosensor. To evaluate the sensing performance, the relative MR change, $\delta R$, is needed to be defined by equation (6).

$$
\delta R=\left|\frac{(\Delta R / R)_{\text {without }}-(\Delta R / R)_{\text {with }}}{(\Delta R / R)_{0}}\right| \times 100(\%)=\frac{1}{2}\left|\sin \theta_{f, \text { without }}-\sin _{f, \text { with }}\right| \times 100(\%)
$$

Where $(\Delta R / R)_{\text {with }}$ indicates the MR ratio of GMR biosensors due to the nanoparticle sensor agents immobilized on the surface of $\mathrm{FL},(\Delta R / R)_{\text {without }}$ indicates the MR ratio without nanoparticle, especially SPNSA, on the surface of FL. Moreover, in equation (6), the $\theta_{f, \text { without }}$ is the angle between FL magnetization and EA when no sensor agent is on the sensor surface and the $\theta_{f, \text { with }}$ is the angle between FL magnetization and EA when the sensor agent is on the sensor surface. For FNSA, the $\theta_{f, w i t h o u t}$ is zero, thus $\delta \mathrm{R}$ can be written by $\delta R=0.5 \sin \theta_{f, \text { with }}$. For SPNSA, the $\theta_{f, \text { without }}$ is not zero due to the excitation field (see Fig. 1(b)) and thus the $\delta \mathrm{R}$ can be rewritten by $0.5 \cos \theta_{f} \cdot d \theta$, where, $d \theta$ is the angle difference before and after the SPNSA captured on the sensor surface $\left(d \theta=\theta_{f, \text { without }}-\theta_{f, \text { with }}\right)$. The fringe field from the FL of GMR biosensors is also considered to affect the magnetic properties of nanoparticle sensor agent in both magnetization direction and magnetic moment due to the magnetic dipole interaction between the FL and the nanoparticles (see Fig. 1-(b)). 
Accordingly, the effect of fringe field from the FL on the $\delta R$ is included in this model to precisely interpret the sensing performance. To find out how many percentages the FL fringe field would influence on the magnetic moment of the magnetic nanoparticles, the interaction factor (IF) defined as $\alpha=\left(1-m^{\prime} \cos \beta / m\right) \cdot 100(\%)$ is employed, where $m^{\prime}$ is the magnetic moment considering the effect of the FL fringe field, $m$ is the original magnetic moment and $\beta$ is the angle difference between the original magnetization of sensor agent and the rotated magnetization due to the fringe field from FL as shown in Fig. 1-(c). In this model, the FNSA is considered as a $\mathrm{CoFe}_{2} \mathrm{O}_{4}$ nanoparticle. It has a remnant magnetization of $22 \mathrm{emu} / \mathrm{g}$ and a diameter of $26 \mathrm{~nm}$ (see Fig. 2-(a) and (b)). The magnetic moment of the $\mathrm{CoFe}_{2} \mathrm{O}_{4}$ nanoparticle is calculated using $\hat{m}=4 \pi M_{r} V$, where $M_{r}$ is the remnant magnetization and $\mathrm{V}$ is the volume of the $\mathrm{CoFe}_{2} \mathrm{O}_{4}$ nanoparticle. Since the $\mathrm{CoFe}_{2} \mathrm{O}_{4}$ nanoparticle has a large remnant magnetization, the excitation field $\left(H_{t}\right)$ is not required, thus the equation (3) can be simplified by equation (7),

$$
E=K_{u} \sin ^{2} \theta_{f}+\frac{1}{2}\left(N_{z}-N_{x}\right) M_{f} \sin ^{2} \theta_{f}-H_{x} M_{f} \cos \theta_{f}-H_{y} M_{f} \sin \theta_{f}-H_{b} M_{f} \cos \theta_{f}
$$

Due to the fringe field from the FL of GMR biosensor $(\Delta H)$, the induced magnetic moment of the $\mathrm{CoFe}_{2} \mathrm{O}_{4}$ nanoparticle becomes $\Delta m=\chi_{f} \cdot \Delta H$, where $\chi_{f}$ is considered as constant because the fringe field is relatively small. In addition, by considering the single domain state of $\mathrm{CoFe}_{2} \mathrm{O}_{4}$ nanoparticle agent, the magnetization direction of the $\mathrm{CoFe}_{2} \mathrm{O}_{4}$ nanoparticle can be calculated using the "Stoner- Wolfarth model" as below:

$$
\begin{aligned}
& E=\frac{1}{2}\left(H_{u}+H_{d}\right) m^{\prime} \sin ^{2} \beta-\Delta H_{x} m^{\prime} \cos \beta-\Delta H_{y} m^{\prime} \sin \beta-H_{b} m^{\prime} \cos \beta \\
& \Delta H_{x}=\frac{M_{f} l w t \cos \theta_{f}}{(h+t / 2+D / 2)^{3}}, \Delta H_{y}=\frac{M_{f} l w t \sin \theta_{f}}{(h+t / 2+D / 2)^{3}}
\end{aligned}
$$

where $m^{\prime}=m+\Delta m$, and $\Delta H_{x}$, and $\Delta H_{y}$ are the longitudinal, and transverse component of the FL fringe field, respectively. As the saturation magnetization of the $\mathrm{CoFe}_{2} \mathrm{O}_{4}$ nanoparticle sensor agent is almost the same as the bulk $\mathrm{CoFe}_{2} \mathrm{O}_{4}$ ferrite, the anisotropy constant, $\mathrm{K}_{1}$, of the sensor agent is assumed as bulk value of $\mathrm{CoFe}_{2} \mathrm{O}_{4}$ ferrite, which is a $2 \times 10^{6} \mathrm{erg} / \mathrm{cm}^{3}$ and thus $H_{u}=2 K_{1} / \mathrm{m}^{\prime}$. The demagnetizing factor is considered as $4 \pi / 3$ thus, $H_{d}=m^{\prime} \cdot 4 \pi / 3$.

The SPN used in this model is also a single $\mathrm{CoFe}_{2} \mathrm{O}_{4}$ nanoparticle but the diameter is a $7 \mathrm{~nm}$ as shown in Fig. 2-(c) and (d). As can be seen in the inset of Fig. 2-(c), the magnetic susceptibility, $X$, of the superparamagnetic $\mathrm{CoFe}_{2} \mathrm{O}_{4}$ nanoparticle is almost constant at a 0.041 independent of applied magnetic field. Using the experimentally obtained $\chi$ value, the magnetic moment of the superparamagnetic $\mathrm{CoFe}_{2} \mathrm{O}_{4}$ nanoparticle under the $H_{b}$ and the $H_{t}$ is determined at $\hat{m}=4 \pi r^{3} \chi H_{b} \bar{x} / 3+4 \pi r^{3} \chi H_{t} \bar{z} / 3$ and the total free energy can be correspondingly re-written by equation (9).

$$
\begin{aligned}
& E=K_{u} \sin ^{2} \theta_{f}+\frac{1}{2}\left(N_{z}-N_{x}\right) M_{f} \sin ^{2} \theta_{f}-H_{x} M_{f} \cos \theta_{f}-H_{y} M_{f} \sin \theta_{f}-H_{b} M_{f} \cos \theta_{f} \\
& -H_{t} M_{f} \sin \theta_{f}
\end{aligned}
$$


As the $X$ value is constant and there is no magnetic anisotropy energy under no excitation field, the IF for the superparamagnetic $\mathrm{CoFe}_{2} \mathrm{O}_{4}$ nanoparticle sensor agent can be simplified as $\alpha_{y}=\Delta H_{y} / H_{t}$, where $\Delta H_{y}$ is the y component of the FL fringe field from GMR biosensor.

$$
\alpha=\frac{M_{s} l w t \sin \theta_{f}}{(h+t / 2+D / 2)^{3} H_{t}} \times 100(\%)
$$

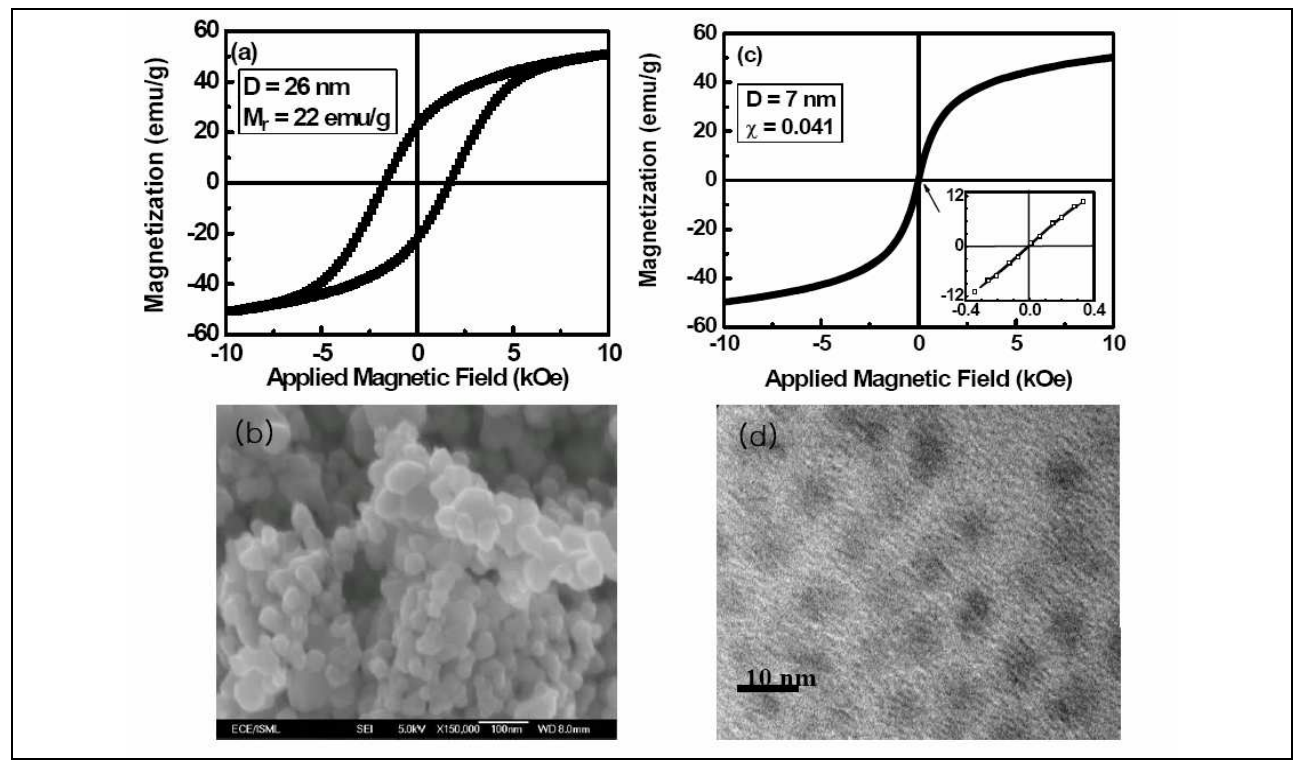

Fig. 2. (a) The hysteresis loop of $\mathrm{CoFe}_{2} \mathrm{O}_{4}$ FNSA with a $26 \mathrm{~nm}$ particle size, and (b) the SEM (Scanning Electron Microscopy) image of the $\mathrm{CoFe}_{2} \mathrm{O}_{4}$ FNSA, (c) the hysteresis loop of $\mathrm{CoFe}_{2} \mathrm{O}_{4}$ SPNSA with a $7 \mathrm{~nm}$ particle size. The inset shows the minor hysteresis loop measured at a $\pm 300 \mathrm{Oe}$, and (d) the TEM (Transmission Electron Microscopy) image of the $\mathrm{CoFe}_{2} \mathrm{O}_{4}$ SPNSA.

\subsection{Comparison of sensing output performance}

Based on the physical model developed in section 2.1, the sensing output performance of an in-vitro GMR biosensor with a single immobilized FNSA or SPNSA is calculated and compared. Figure 3 shows the dependence of sensor width at the fixed sensor geometry (sensor aspect ratio) on the $\delta \mathrm{R}$ and the IF of the in-vitro GMR biosensors. The sensor width, $\mathrm{W}$, of the GMR biosensor is changed from 10 to $80 \mathrm{~nm}$ at the different aspect ratio $(\mathrm{L}: \mathrm{W})$ changed from $3: 1$ to $10: 1$. The purpose of changing the sensor aspect ratio is to explore the effects of vortex magnetization on the surface of FL due to the geometrically-induced demagnetizing factor (Girgis et al., 2000). The $\mathrm{H}_{\mathrm{b}}$, and the $\mathrm{h}$ are fixed at a $50 \mathrm{Oe}$, and a 30 $\mathrm{nm}$, resepctively for precise comparison. The $\mathrm{CoFe}_{2} \mathrm{O}_{4}$ FNSA, and SPNSA has a mean particle size of $26 \mathrm{~nm}$ and $7 \mathrm{~nm}$, resepctively. The $\delta \mathrm{R}$ and its variation due to the change of $\mathrm{W}$ is numerically analyzed by considering both the "effective sensing area", which is defined as the area formed on the FL surface whose magnetic spins can be coherently 
rotated by the stray magnetic field induced by the sensor agent, and the development of "inactive sensing area", which is not responded by the stray field, due to the increase of IF induced by the geometrically-increased magnetic anisotropy of FL.

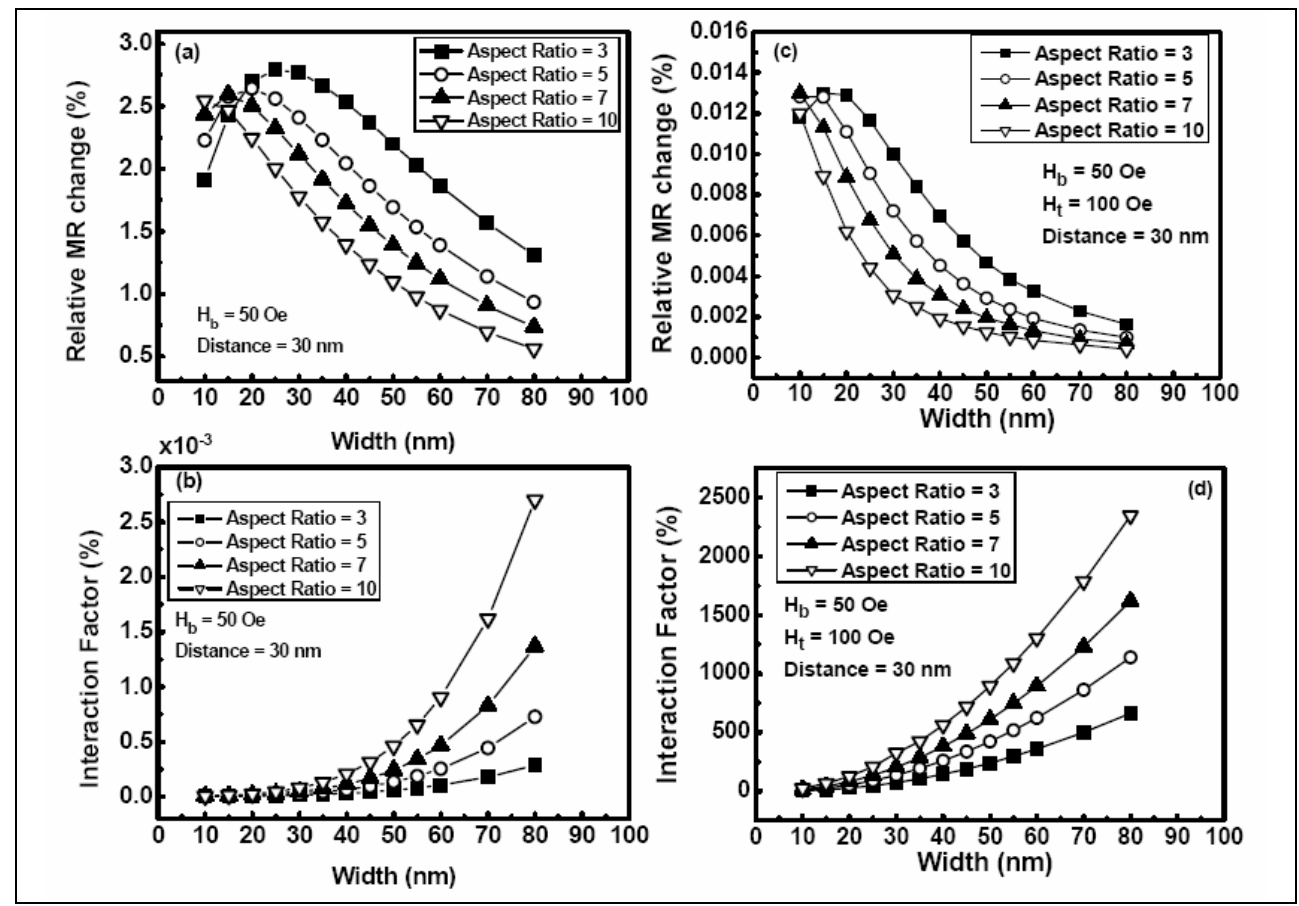

Fig. 3. The physical dependence of sensor width, $W$, on the relative $M R, \delta R$, and the interaction factor, IF, (a) $\delta$ R, GMR biosensor with a FNSA, (b) IF, GMR biosensor with a FNSA, (c) $\delta R$, GMR biosensor with a SPNSA, and (d) IF, GMR biosensor with a SPNSA.

As shown in Fig. 3, the in-vitro GMR biosensors with an immobilized single FNSA or SPNSA exhibit the same physical characteristics that the $\delta \mathrm{R}$ is abruptly decreased above the maximized value obtained at the optimized sensor width, $\mathrm{W}_{\mathrm{op}}$, and that the IF is almost squarely increased, by increasing the $\mathrm{W}$ as well as the aspect ratio. This is supposed to be due to the increase of "inactive sensing area" and the magnetic anisotropy of FL induced by the increased sensor size proportional to the W. However, it is clearly noted that the absolute $\delta \mathrm{R}$ and IF values of the in-vitro GMR biosensor with a FNSA are much larger than those with a SPNSA. As can be clearly seen in Fig. 3-(a) and (c), the $\delta$ R obtained from the invitro GMR biosensor with an aspect ratio of 3: $1\left(75 \mathrm{~nm}(\mathrm{~L}) \times 25 \mathrm{~nm}\left(\mathrm{~W}_{\mathrm{op}}\right)\right)$ and an immobilized FNSA is a $2.72 \%$, while the $\delta \mathrm{R}$ for the in-vitro GMR biosensor with a SPNSA, which has the same aspect ratio $\left(45 \mathrm{~nm}(\mathrm{~L}) \times 15 \mathrm{~nm}\left(\mathrm{~W}_{\mathrm{op}}\right)\right)$, is a $0.013 \%$. In addition, the variation of IF values depending on the $\mathrm{W}$ of the in-vitro GMR biosensor with a FNSA is negligibly small compared to those with a SPNSA as shown in Fig. 3-(b) and (d). The practically allowable sensor size based on the physical limit of current sensor fabrication technology, especially nanoelectronics technology, is another physical parameter to be considered in evaluating the sensing performance. Considering the patterning limit of EBL 
(Electron Beam Lithography) technique (> $50 \mathrm{~nm}$ ) and the geometrically-induced demagnetizing factor of FL directly relevant to the sensor aspect ratio and the IF, the minimum sensor size can be determined in the range between $150 \mathrm{~nm}(\mathrm{~L}) \times 50 \mathrm{~nm}(\mathrm{~W})$ and $250(\mathrm{~nm}) \times 50 \mathrm{~nm}(\mathrm{~W})$. However, as verified in Fig. 3-(a) and (c), the $\delta \mathrm{R}$ values obtained from these sizes of in-vitro GMR biosensor with a SPNSA are too small to be considered for a real biosensor application.

According to the numerically analyzed sensing performance summarized in Fig. 3, it is clearly demonstrated that an in-vitro GMR biosensor with an immobilized single $\mathrm{CoFe}_{2} \mathrm{O}_{4}$ FNSA is more suitable for SMD due to its higher $\delta \mathrm{R}$, less IF dependence, and practically allowable sensor size. The large remnant magnetization of single $\mathrm{CoFe}_{2} \mathrm{O}_{4}$ FNSA allowing to produce a sufficiently large stray field and to maintain extremly small variation of IF is the main physical reason for the technical promise of GMR biosensor with an immobilized single FNSA for SMD.

\section{Optimizing the sensor geometry of an in-vitro GMR biosensor with an immobilized FNSA for SMD}

In this chapter, the detailed spatial magnetic field interactions between the single $\mathrm{CoFe}_{2} \mathrm{O}_{4}$ FNSA and the FL of an in-vitro GMR biosensor is numerically analyzed to predict the optimized sensor geometry that maximizes the sensng perfromance for SMD prior to fabrication. In order to more accurately analyze the spatial magentic field interactions on the FL surface, the longitudinal and the transverse components of the stray field produced by the FNSA are considered. The optimized sensor geometry at a given remnant magentic moment of the FNSA is predicted by evaluating the "effective sensing area". The optimized sensor geometry is expressed in terms of the effective distance $(\delta)$, which includes the radius, $a$, of FNSA, the length of biological entities (especially, DNA including probe), membrane thickness, and the passivation layer, as well as the critical sensor length $\left(l_{c}\right)$, and the critical sensor width $\left(\mathrm{w}_{\mathrm{c}}\right)$. The experimentally demonstrated sensing performance of an in-vitro GMR biosensor with an immobilized $\mathrm{CoFe}_{2} \mathrm{O}_{4}$ FNSA is also compared to the numerically calculated sensing performance to confirm the effectiveness of the physical model introduced in this chapter.

\subsection{Analytical model for optimizing sensor geometry and geometrical parameters}

Figure 4 shows the schematic diagram of an in-vitro GMR biosensor with an immobilized single FNSA (a) and the typical MR curve (b) obtained from the $\mathrm{Si} / \mathrm{Ta} / \mathrm{Ni}_{80} \mathrm{Fe}_{20} / \mathrm{Ir}_{22} \mathrm{Mn}_{78} /$ $\mathrm{Co}_{84} \mathrm{Fe}_{16} / \mathrm{Ru} / \mathrm{Co}_{84} \mathrm{Fe}_{16} / \mathrm{Cu} / \mathrm{Co}_{84} \mathrm{Fe}_{16} / \mathrm{Ni}_{80} \mathrm{Fe}_{20} / \mathrm{Ta}$ exchange biased synthetic GMR spinvalve biosensor. For the numerical calculation, it is assumed that the $\mathrm{CoFe}_{2} \mathrm{O}_{4}$ FNSA has an $a$ $=250 \mathrm{~nm}$, and a mass density of $5.29 \mathrm{~g} / \mathrm{cm}^{3}$ (Lee et al., 2007). By considering only the logitudinal field compoent of the stray field produced by the immobilized single $\mathrm{CoFe}_{2} \mathrm{O}_{4}$ FNSA, $B_{x}$ on the surface of FL along the $x$ and $y$ axis from equation (2) is simplified by equation (11) (Schepper et al., 2006).

$$
\begin{aligned}
& B_{x, x-\text { axis }}=m \cdot \frac{2 x^{2}-z^{2}}{\left(x^{2}+z^{2}\right)^{5 / 2}} \\
& B_{x, y \text {-axis }}=m \cdot \frac{-y^{2}-z^{2}}{\left(y^{2}+z^{2}\right)^{5 / 2}}
\end{aligned}
$$


The calculated magnetic field distribution and the two geometrically critical parameters, which are essential to determine the optimized sensor geometry, are also denoted in Fig. 4(a). The geometrical parameters of the in-vitro GMR biosensor with an immobilized single FNSA are first determined by considering the longitudinal component of the stray field. The effective magnetization, $\beta$, is defined as the ratio of the total magnetization of the $\mathrm{CoFe}_{2} \mathrm{O}_{4}$ FNSA to the longitudinal field component of the stray field, $\beta=m / B_{x}$. The $\delta$ is defined as $\delta=h+a$. The geomtrical parameters, the $\mathrm{l}_{\mathrm{c}}$, and the $\mathrm{w}_{\mathrm{c}}$ for achieving the optimized sensor geometry, which maximize the sensor output performance, are dependent on $\beta$ and $\delta$. These geometrical parameters, which determine the "effective sensing area", $l_{c} \times w_{c}$, are derived from equation (11) by considering $\mathrm{x}$ and $\mathrm{y}$ at the points where $\mathrm{B}_{\mathrm{x}}$ is equal to the sensor switching field, $\mathrm{B}_{\mathrm{sw}}$ (with $\beta_{s w} \equiv m / B_{x}$ ). The finally determined $\mathrm{l}_{\mathrm{c}}$, and $\mathrm{w}_{\mathrm{c}}$ are given by,

$$
\begin{aligned}
& l_{c}=|2 x|=2 \sqrt{2} \delta \sqrt{\frac{\beta_{s w}-\delta^{3}}{4 \beta_{s w}+5 \delta^{3}}} \\
& w_{c}=|2 y|=2 \sqrt{\beta_{s w}^{2 / 3}-\delta^{2}}
\end{aligned}
$$

The insert in Fig. 4-(b) highlights the two characteristic parameters relevant to the operation of the in-vitro GMR biosensor; the $\mathrm{B}_{\mathrm{sw}}$ and the detectable field limit $\left(\mathrm{B}_{\mathrm{DL}}\right)$ directly associated with the exchange bias field of the in-vitro GMR biosensor, are defined in terms of the intensity of stray field produced by the single $\mathrm{CoFe}_{2} \mathrm{O}_{4}$ FNSA. The critical effective distance, $\delta_{\mathrm{c}}$, can be obtained by considering the operating conditions of the GMR biosensor including $\mathrm{B}_{\mathrm{sw}}, \mathrm{B}_{\mathrm{DL}}$, and the $\mathrm{M}_{\mathrm{r}}$ of the single $\mathrm{CoFe}_{2} \mathrm{O}_{4}$ FNSA. If $\mathrm{B}_{\mathrm{x}}$ is in the sensor operating range, $B_{\mathrm{sw}}<\mathrm{B}_{\mathrm{x}}<\mathrm{B}_{\mathrm{DL}}$, $\delta$ can be expressed as a function of $\beta$. On the other hand, if $\mathrm{B}_{\mathrm{x}}$ is smaller than $\mathrm{B}_{\mathrm{sw}}\left(\mathrm{B}_{\mathrm{sw}}>\mathrm{B}_{\mathrm{x}}\right)$, then $l_{c}=w_{c}=0$. Thus, the critical effective distance, $\delta_{\mathrm{c}}$, for the sensor

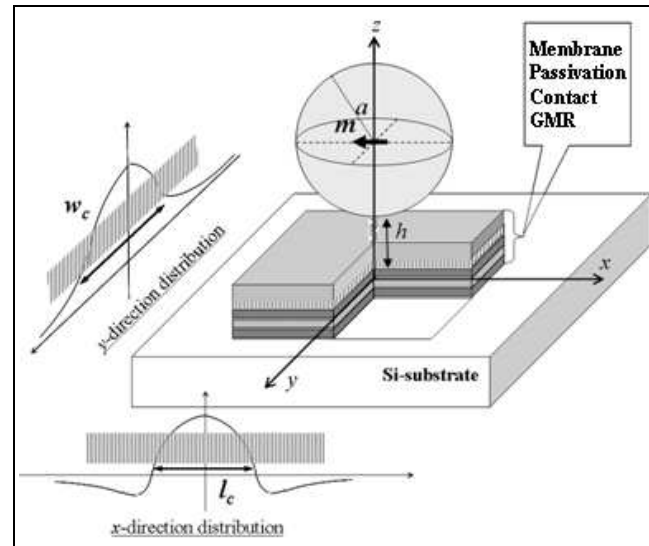

(a)

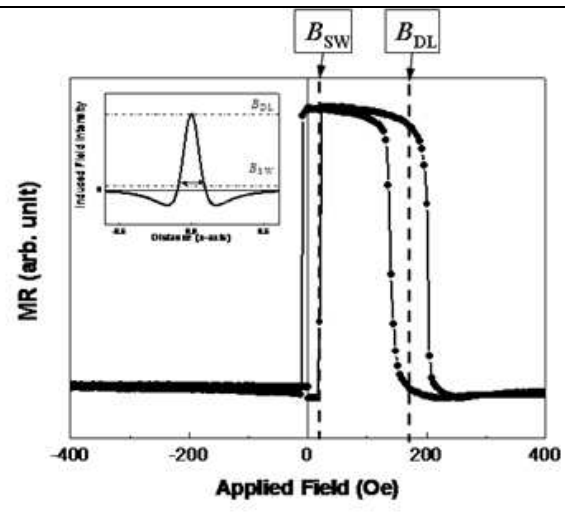

(b)

Fig. 4. (a) A schematic diagram of in-vitro GMR biosensor with an immobilized single FNSA, the field distribution, and the definition of geometrical parameters considering for optimizing sensor geometry, and (b) a typical MR curve of GMR biosensor and the definition of two sensing characteristics parameters. 
operation based on the non-switching conditions: $B_{s w}>B_{x}$, and equation (12) can be determined at $\delta_{c}=\sqrt[3]{\beta}$. In addition, from equation (12), the aspect ratio, $w_{c} / l_{c}$ for the optimized sensor geometry can be expressed by equation (13).

$$
w_{c} / l_{c}=\frac{2 \sqrt{\beta_{s w}^{2 / 3}-\delta^{2}}}{2 \sqrt{2} \delta \cdot \sqrt{\frac{\beta_{s w}-\delta^{3}}{4 \beta_{s w}+5 \delta^{3}}}}=\sqrt{\frac{\left(\beta_{s w}^{2 / 3}-\delta^{2}\right) \cdot\left(4 \beta_{s w}+5 \delta^{3}\right)}{2 \delta^{2}\left(\beta_{s w}-\delta^{3}\right)}}
$$

The numerically analyzed magnetic field distribution on the surface of the FL finally obtained by equation (13) clearly demonstrates that the optimized geometrical parameters, $1_{c}$, and $w_{c}$ are directly relevant to $\delta$ and $\beta_{s w}$. In order to more accurately predict the optimized sensor geometry based on the "effective sensing area, $l_{c} \times w_{c}$ ", the numerical calculation is extended to two dimensional field component, both longitudinal and transverse field components, on the FL surface. The "Stoner- Wolfarth model" (or the "asteroid curve model") is employed for the detailed calculation (Hirota et al., 2002).

\subsection{Optimizing the sensor geometry considering the one dimensional (longitudinal) field component}

As described in the analytical model developed in section 3.1, the optimization of sensor geometry with an immobilized $\mathrm{CoFe}_{2} \mathrm{O}_{4}$ FNSA is based on the determination of $1_{c}$, and $\mathrm{w}_{\mathrm{c}}$ by considering the longitudinal field component of $\mathrm{B}_{\mathrm{x}}$, and $\mathrm{B}_{\mathrm{y}}$, on the FL surface. Figure 5 shows the contour diagrams of the magnetic field intensity and its distributions, $\mathrm{B}_{\mathrm{x}}$, and $\mathrm{B}_{\mathrm{y}}$ on the FL surface as a function of $\delta$ (for $\delta=0.5,1.0$, and $2.0 \mu \mathrm{m}$ ). As can be seen in Figs. 5-(a), 5-(c), and 5(e), the maximum $B_{x}$ is rapidly decreased from 691.2 to $10.8 \mathrm{G}$ by increasing $\delta$ from 0.5 to 2.0 $\mu \mathrm{m}$. As shown in Fig. 4-(b), the in-vitro GMR biosensor considered in this model is operated at magnetic field intensity in the range from $12 \mathrm{G}\left(\mathrm{B}_{\mathrm{sw}}\right)$ to $176 \mathrm{G}\left(\mathrm{B}_{\mathrm{DL}}\right)$. Considering these the magnetic characteristics of GMR biosensor, the shaded region observed at $\delta=0.5 \mu \mathrm{m}$ due to the large field intensity (Fig. 5-(a)) and all the regions shown in Fig. 5-(e) do not contribute to the sensor operation. This indicates that the $l_{c}$ and the $w_{c}$ for the optimized sensor geometry based on equation (12) should be determined at $\delta_{\mathrm{c}}<0.79 \mu \mathrm{m}$, which corresponds to the sensor operating condition of $B_{x} \leq B_{D L}$. Furthermore, by combining the calculated value of $\delta_{c}$ with the physical parameters of single $\mathrm{CoFe}_{2} \mathrm{O}_{4}$ FNSA and equation (12), the $1_{c}$, and the $\mathrm{w}_{\mathrm{c}}$ are determined to be $\sim 1.12 \mu \mathrm{m}$, and $\sim 3.52 \mu \mathrm{m}$. Based on the numerical calculation, the aspect ratio $\left(w_{c} / l_{c}\right)$ for the optimized sensor geometry of in-vitro GMR biosensor with an immobilized single $\mathrm{CoFe}_{2} \mathrm{O}_{4}$ FNSA $(a=250 \mathrm{~nm})$ is determied at $w_{c} / l_{c}=3.14$.

The calculation results shown in Fig. 5 clearly demonstrates that the geometrical and systematic design parameters $\left(\delta, l_{c}\right.$, and $\left.w_{c}\right)$ of the in-vitro GMR biosensor for producing a highly stable sensing performance can be precisely predicted prior to fabrication if the remnant magnetization of the single $\mathrm{CoFe}_{2} \mathrm{O}_{4}$ FNSA and the GMR characteristics of the sensor are known.

\subsection{Optimizing the sensor geometry considering the longitudinal and transverse field components}

Dependence of $\delta$ on the transverse component, $\mathrm{B}_{\mathrm{y}}$, is also estimated to confirm its physical contribution to the optimization of in-vitro GMR biosensor geometry. Figure 5-(b), 5-(d), 

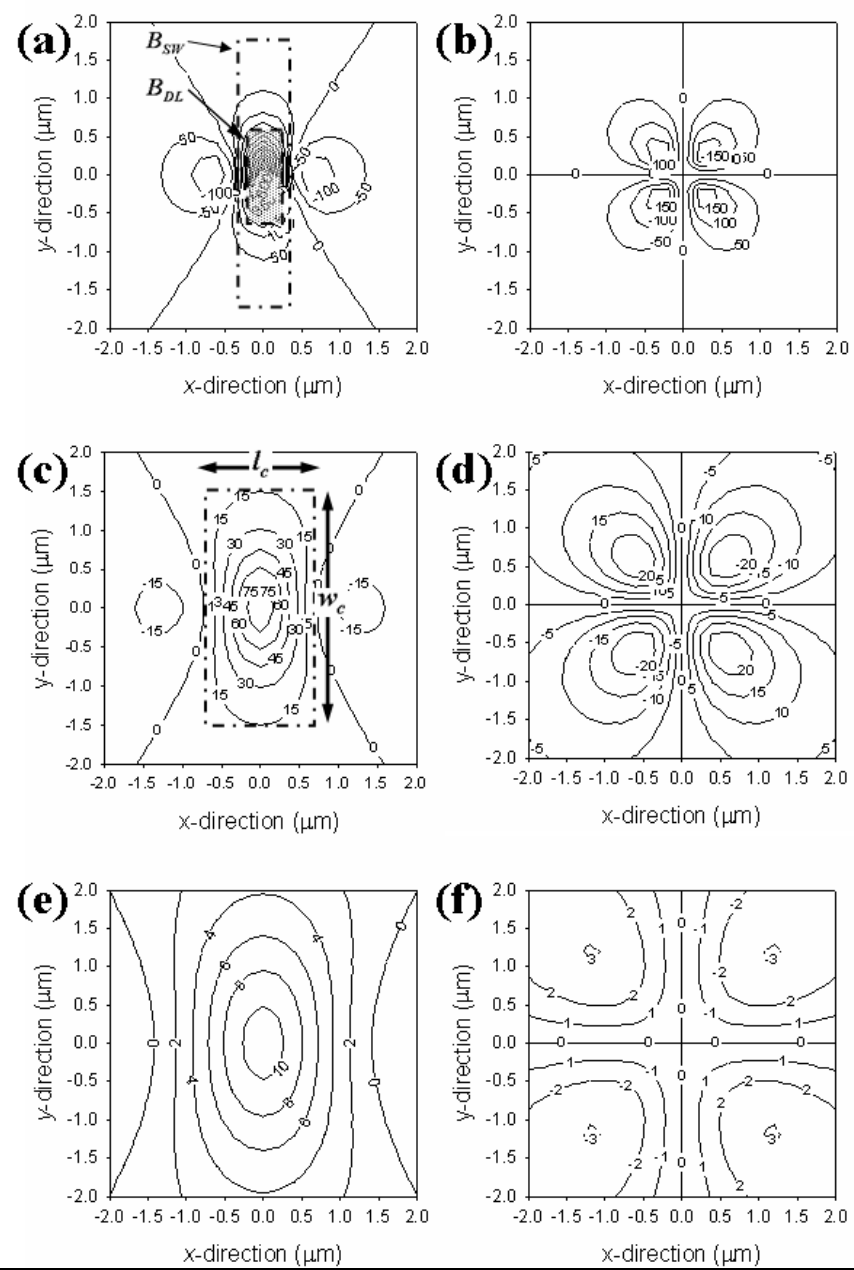

Fig. 5. Calculated contour diagrams of the longitudinal (left column) and transverse (right column) components of the magnetic field produced by an immobilized $\mathrm{CoFe}_{2} \mathrm{O}_{4} \mathrm{FNSA}_{\text {on }}$ the FL surface where $\delta$ is varied from 0.5 to $2.0 \mu \mathrm{m}$. The area defined by the dashed-dotted line and the shaded region show the optimized sensor geometry, and the undetectable region, respectively.

and 5-(f) show the contour diagrams of $B_{y}$ as a function of $\delta$ changed from 0.5 to $2.0 \mu \mathrm{m}$. Similar to the calculation results shown in Figs. 5-(a), 5-(c), and 5-(e), the $\mathrm{B}_{\mathrm{y}}$ has a strong dependence on $\delta$. However, the distribution of $B_{y}$ is completely different from $B_{x}$. The distribution of $B_{x}$ on the FL surface shows an ellipsoidal shape with the major axis along the $\mathrm{y}$-axis, while $\mathrm{B}_{\mathrm{y}}$ exhibits a distribution that has a maximum and minimum field intensity of $B_{y, \text { max }} \approx 1 / 3 B_{x, \text { max }}$ at the position of $( \pm \delta, \mp \delta)$. The numerical comparison between $\mathrm{B}_{\mathrm{x}}$ and $\mathrm{B}_{\mathrm{y}}$ depending on $\delta$ suggests that both components of the stray field should be simultaneously 
considered for a more accurate prediction of the sensor geometry. Accordingly, the "StonerWolfarth model": $H_{k}^{2 / 3}=H_{x}^{2 / 3}+H_{y}^{2 / 3}$, is employed to accurately analyze the spatial magnetic field distribution and intensity on the FL surface. Even though the "StonerWolfarth model" assumes that the FL magnetizations are coherently rotated by the stray field and are homogneous across the entire FL surface, this model is considerably useful in interpreting the physical behavior of the in-vitro GMR biosensor under a highly localized magnetic dipole field from the immobilized single $\mathrm{CoFe}_{2} \mathrm{O}_{4}$ FNSA. Figure 6 shows the magentic field distribution and intesity considering both the logitudinal and transverse field components with different effective distances: $\delta=0.5,1.0$, and $2.0 \mu \mathrm{m}$. Unlike the ellipsoidal shape of the "effective sensing area" shown in Fig. 5, the coherently rotated magnetization of the FL induced by two-dimensional magnetic field components shows a more complicated and extended "effective sensing area" due to the contribution of the transverse field component. Figure 7 shows the optimized sensor geometry (white line) and the "effective sensing area" (bright gray region) calculated by considering the one-dimensional

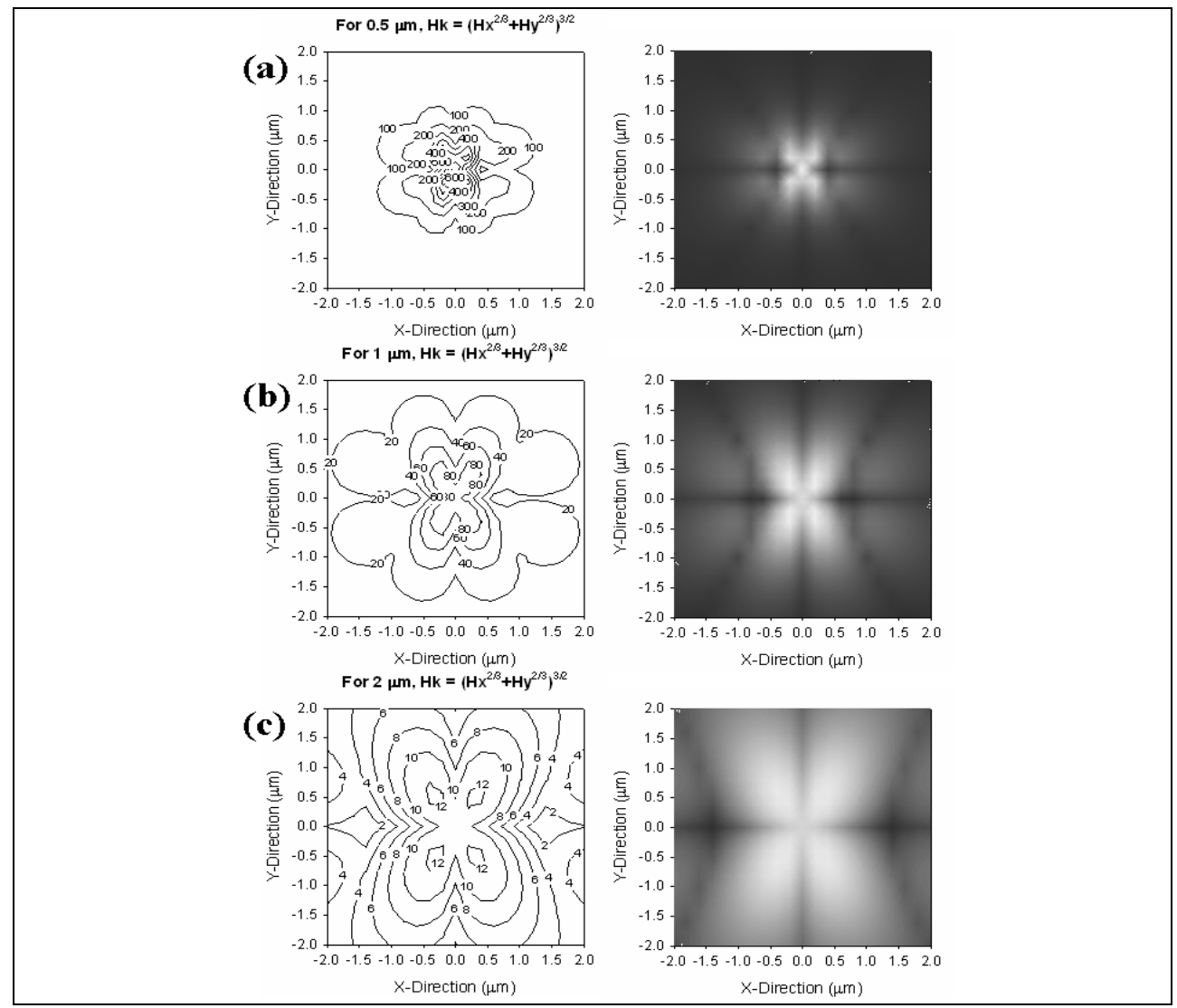

Fig. 6. The magnetic field distribution and intensity on the FL surface calculated by considering the longitudinal and transverse field components at the different effective distance of $\delta$. (a) 0.5 , (b) 1.0, and (c) $2.0 \mu \mathrm{m}$ 
(Fig. 7-(a)) and the two-dimensional components (Fig. 7-(b)) based on the "Stoner- Wolfarth model". Although the numerical values of optimized geometrical parameters determined at the effective distance of $\delta=0.79 \mu \mathrm{m}$ are the same as $l_{c}=1.12 \mu \mathrm{m}$, and $\mathrm{w}_{\mathrm{c}}=3.52 \mu \mathrm{m}$, the "effective sensing area" directly relevant to the sensing output performance is completely different. As can be seen in Fig. 7-(b), the "effective sensing area" is extended due to the transverse field component. This correspondingly results in enhancing the output signal of the in-vitro GMR biosensor. However, as can be also seen in Fig. 7-(b), an undetectable area in the vicinity of center of the optimized sensing area is developed due to the spatial magnetic field interaction. Making a GMR biosensor with a larger exchange bias field and introducing a specially designed sensor structure with a high permeability magnetic shield layer are suggested as an effective solution for the undesirable technical problem.
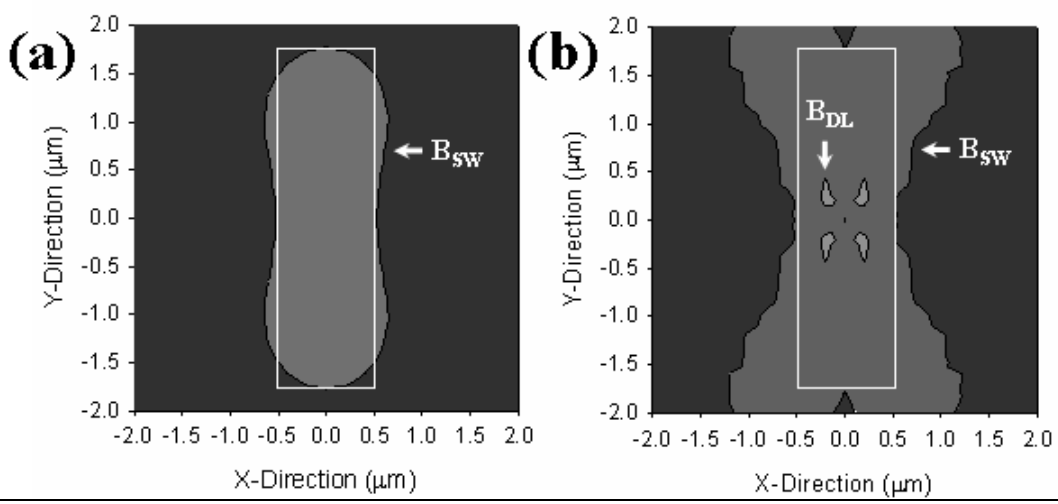

Fig. 7. Comparison of the optimized sensor geometry (square region) and the "effective sensing area" calculated by considering the (a) one-dimensional filed component, and (b) two-dimensional field component on the FL surface.

\subsection{Demonstration of sensing performance of the in-vitro GMR biosensors with optimized sensor geometry}

The sensing performance of an in-vitro GMR biosensor with an immobilized $\mathrm{CoFe}_{2} \mathrm{O}_{4}$ ferrimagentic nanobead SA geomtrically optimized by the analytical model developed in chapter 3.1 is demontrated to confirm its practical effectiveness. The $\mathrm{CoFe}_{2} \mathrm{O}_{4}$ nanobead with a mean raius, $a$, of $925 \mathrm{~nm}$ synthesized by using a modified sol-gel mehtod is considered as a ferrimagentic nanobead SA. The optimized sensor geomtry of the in-vitro GMR biosesnor based on the equations (11) (13) as well as considering a $925 \mathrm{~nm}$ of mean nanobead size is calcuated to determine the "effective sensing area, $l_{c} \times w_{c}$ ". The sensing output performance of the optimized GMR biosensors is evaluated as a function of sensor length, $l$, at the fixed $\mathrm{w}_{\mathrm{c}}$ by controlling the size of $\mathrm{CoFe}_{2} \mathrm{O}_{4}$ nanobead SA, which is systematically varied in the range of $a=925 \mathrm{~nm} \pm 20.5 \%$ as shown in Fig. 8-(a).

The controlled nanobead size leads to changing the $l$ at the fixed $\mathrm{w}_{\mathrm{c}}$ due to the variation of stray field intensity caused by the change of effective distance. The GMR biosensor used for this demosntration has a strucutre of $\mathrm{Si} / \mathrm{Ta}(5) / \mathrm{Ni}_{80} \mathrm{Fe}_{20}(2) / \mathrm{Ir}_{22} \mathrm{Mn}_{78}(20) / \mathrm{Co}_{84} \mathrm{Fe}_{16}(2) /$ $\mathrm{Ru}(0.75) / \mathrm{Co}_{84} \mathrm{Fe}_{16}(2) / \mathrm{Cu}(2.3) / \mathrm{Co}_{84} \mathrm{Fe}_{16}(0.5) / \mathrm{Ni}_{80} \mathrm{Fe}_{20}(2.5) / \mathrm{Ta}(3 \mathrm{~nm})$ and is patterned by using an electron beam lithography (EBL) and a typical photolithography. The patterned 


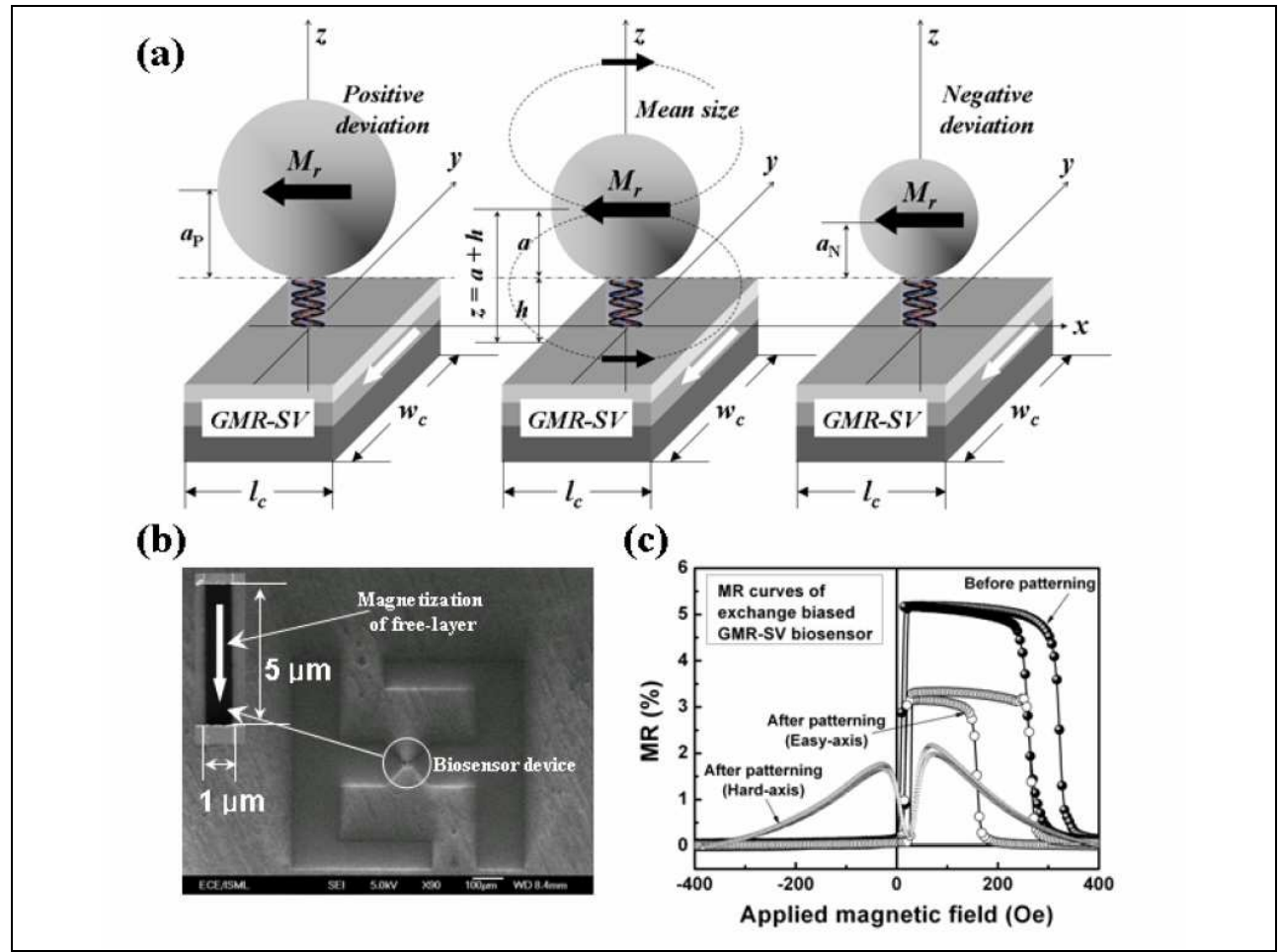

Fig. 8. (a) Schematic diagram of in-vitro GMR biosensors with an immobilized $\mathrm{CoFe}_{2} \mathrm{O}_{4}$ ferrimagentic nanobead SA with different bead sizes controlled in the range of $a=925 \mathrm{~nm} \pm$ $20.5 \%$, (2) the patterned GMR biosensor with the geomtry of $l=1 \mu \mathrm{m}$, and $\mathrm{w}_{\mathrm{c}}=5 \mu \mathrm{m}$, and (c) GMR behaviour.

GMR biosensor structure and its GMR behaviour for the before and after patterning, and for the hard axis response are shown in Figs. 8(b), and (c), respectively. As can be seen in Fig. 8(a), the magnetization of FL is orthogonally coupled to the pinned layer, and the stray field produced by the single $\mathrm{CoFe}_{2} \mathrm{O}_{4}$ nanobead SA is applied to the hard axis of FL magnetization for the detection of output sensing signal.

On the basis of the numerical analysis, the intensity of stray field produced by the $\mathrm{CoFe}_{2} \mathrm{O}_{4}$ nanobead SA with a radius of 750 (-20.5\%, negative standard deviation), 925 (mean nano bead size), and $1150 \mathrm{~nm}(+20.5 \%$, positive standard deviation) is calculated by considering the experimentally obtained $M_{r}$ values to determine the $1_{c}$. The calculated maximum field intensity is a $67.8,116.5$, and $177.1 \mathrm{Oe}(\mathrm{G})$, respectively and the $1_{c}$ is revealed to be a 0.85 , 1.08 , and $1.31 \mu \mathrm{m}$, respectively at the fixed $w_{c}=5 \mu \mathrm{m}$. Figure 9 shows the detected output signal obtained from the in-vitro GMR biosensor shown in Fig. 8-(b). The detected signal is captured by using an oscilloscope. As can be clearly seen in Fig. 9-(a), when a DC magnet with a constant field of 103 Oe $(\mathrm{G})$ is brought proximity to the GMR biosensor, an output signal of $\mathrm{V}_{\text {out }}=6.13 \mathrm{mV}\left(\mathrm{V}_{\text {output }}=613 \mathrm{mV}\right.$ after 100 times amplication using a 741 OP-AMP) is successfully achieved. This is attributed to the MR change of the GMR biosensor, $\Delta R / R_{0}=$ $2.2 \%$, which is exactly equal to the maximum MR ratio obtained along the hard-axis of the 
patternd GMR biosensor shown in Fig. 8-(c). At a 103 Oe (G) of field intensity, the "effective sensing area", $l_{c} \times w_{c}$ induced by the DC magnetic field intensity is larger than the patterned sensor geomtry of $l=1 \mu \mathrm{m}$, and $w=5 \mu \mathrm{m}$. This indicates that all the FL magnetizations are fully rotated by the applied DC magnetic field resulting in exhibiting the maximum MR ratio of $2.2 \%$. In contrast, the output signals obtained from the in-vitro GMR biosensors activated by the $\mathrm{CoFe}_{2} \mathrm{O}_{4}$ nanobead SAs show a strong dependence on the size of nanobead SA. As can be seen in Figs. 9-(b), (c), and (d), the output voltage and the $\Delta \mathrm{V}_{\text {out }} / \mathrm{V}$ of the GMR biosensor activated by the $\mathrm{CoFe}_{2} \mathrm{O}_{4}$ nanobead SA with a size of 750, 925, and $1150 \mathrm{~nm}$ are $\mathrm{V}_{\text {out }}=6.07 \mathrm{mV}\left(\Delta \mathrm{V}_{\text {out }} / \mathrm{V}=1.2 \%\right), \mathrm{V}_{\text {out }}=6.12 \mathrm{mV}\left(\Delta \mathrm{V}_{\text {out }} / \mathrm{V}=2.0 \%\right)$, and $\mathrm{V}_{\text {out }}=$ $6.10 \mathrm{mV}\left(\Delta \mathrm{V}_{\text {out }} / \mathrm{V}=1.7 \%\right)$, respectively. Even though the non-uniformity and the position dependent stray field intensity produced by the nanobead SA can be considered to be partially influenced on the variation of output sensing signal, the observed sensing signal depending on the size of nanobead SA is primarily interpreted in terms of two physical parameters: (a) the change of stray field intensity relevant to the switching field, and (b) the "inactive sensing area" as well as the development of "undetectable sensing area". As can be

(a)

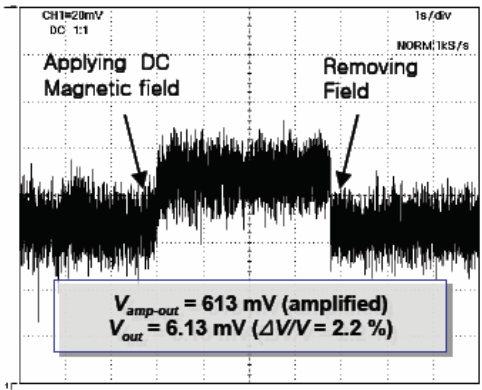

$1.85 \mu \mathrm{m}$ particle (Mean particle size)

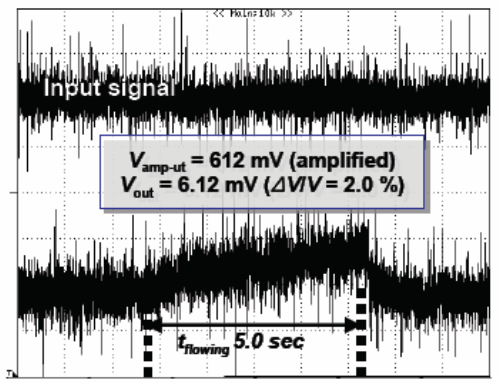

(b)

$1.5 \mu \mathrm{m}$ particle (Negative; $1.85 \mu \mathrm{m}-20.5 \%$ )

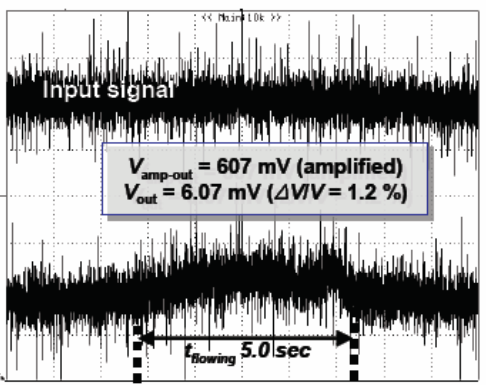

$2.3 \mu \mathrm{m}$ particle (Positive; $1.85 \mu \mathrm{m}+\mathbf{2 0 . 5} \%$ )

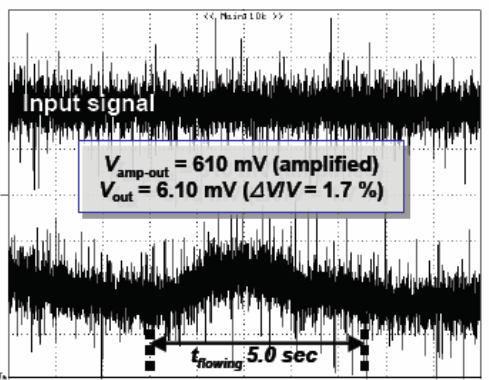

Fig. 9. Output sensing signal captured from the in-vitro GMR biosensor with geometry of $l=$ $1 \mu \mathrm{m}$ and $w=5 \mu \mathrm{m}$. (a) activated by DC magnet, (b) activated by $750 \mathrm{~nm}$ size $\mathrm{CoFe}_{2} \mathrm{O}_{4}$ nanobead SA, (c) activated by $925 \mathrm{~nm}$ size $\mathrm{CoFe}_{2} \mathrm{O}_{4}$ nanobead SA, and (d) activated by 1150 nm size $\mathrm{CoFe}_{2} \mathrm{O}_{4}$ nanobead SA. 
confirmed from the calcualtion results, the $0.85 \mu \mathrm{m}$ of $l_{c}$ determined by the $750 \mathrm{~nm}$ size of nanobead SA at the fixed $w_{c}=5 \mu \mathrm{m}$ is smaller than the geometry of patterned GMR biosensor, $l=1 \mu \mathrm{m}$ and $w=5 \mu \mathrm{m}$, that results in the reduction of MR ratio due to the "inactive sensing area". In addition, the reduced stray field intensity due to the decrease of nanobead size leads to the reduction of switching field that results in a lower output sensing signal as shown in Fig. 8-(c). The slight decrease of output sensing signal obtained from the GMR biosensor with an $1150 \mathrm{~nm}$ size nanobead SA is thought to be due to the development of "undetectable sensing area" as shown in Fig. 7-(b). The large stray field intensity, around 177.1 Oe $(\mathrm{G})$, obtained from the large size of nanobead SA is comparable to the exchange bias field of the patterned GMR biosensor. This induces the partial magnetic reversal of pinned layer resulting in a slight MR degradation. Furthermore, the spatial magnetic field interaction due to the large stray field intensity causes to form an undesirable "undetectable sensing area" at the central region of FL surface of the patterned GMR biosensor that leads to the reduction of MR ratio as well as the output sensing signal.

\section{Effects of a specially designed magnetic shield layer (MSL) on the sensing performance of an in-vitro GMR biosensor with an immobilized single FNSA for SMD}

As previously discussed, the in-vitro GMR biosensor with an immobilized single $\mathrm{CoFe}_{2} \mathrm{O}_{4}$ FNSA is suitable for SMD. However, accoridng to the numerical analysis on the spatial field interaction of the stray field, which is produced by a single FNSA, on the FL surface, the field distribution is found to be so complicated and non-uniform that it can not be easily interpreted. In particular, the creation of "undershoot field regions", which are formed at both edges of the maximum field intensity regions as well as the central regions of FL surface resulted from the spatial magnetic field interaction, is revealed as the most severe problem to induce the reduction of output sensing signal and the sensing stability of the invitro GMR biosensor. Thus, a new sensor structure, which can solve this technical challenge, is urgently required in a molecular based diagnostic GMR biosensor system for achieving more stable SMD.

In this chapter, a new structure of in-vitro GMR biosensor with a specially designed magnetic shield layer (MSL) is introduced and discussed based on the numerically analyzed calculation results to explore its effectiveness for the improvement of sensing performance as well as the sensing stability. The effects of MSL thickness including magnetic permeability of MSL, and gap width of MSL on the change of "undershoot field" as well as the "stray field intensity" are primarily discussed to demonstrate the physical contribution of MSL to the sensing performance of the in-vitro GMR biosensor considering for SMD.

\subsection{Designing of in-vitro GMR biosensor with magnetic shield layer (MSL)}

Figure 10-(a) shows a schematic diagram of an in-vitro GMR biosensor with a specially designed MSL. As shown in Fig. 10-(a), the in-vitro GMR biosensor has a single $\mathrm{CoFe}_{2} \mathrm{O}_{4}$ FNSA immobilized on the FL surface with a distanace of $h \mu \mathrm{m}$, which is defined as the distacne between the FNSA and the FL surface of the GMR bionsensor. Accoridng to the physical model nuemrically developed in chapter 3.1 (Schepper et al., 2006), the $h$ can be expressed as equation (14), 


$$
\begin{aligned}
& h=\left(4 \pi \times m \frac{1}{B_{D L}}\right)^{1 / 3}-a \\
& m=\frac{4 \pi}{3} a^{3} M_{r}
\end{aligned}
$$

In this numerical calculation, an IrMn based exchange biased GMR spin-valve device is considered as a sensing element. Thus, to achieve a stable sensing performance, the maximum field intensity produced by the single FNSA should be adjusted to be lower than the exchange bias field of the GMR biosensor. Considering this sensor operating condition, $\mathrm{B}_{\mathrm{DL}}$ is determined based on the experimentally obtained exchange bias field from the patterned GMR biosensor.

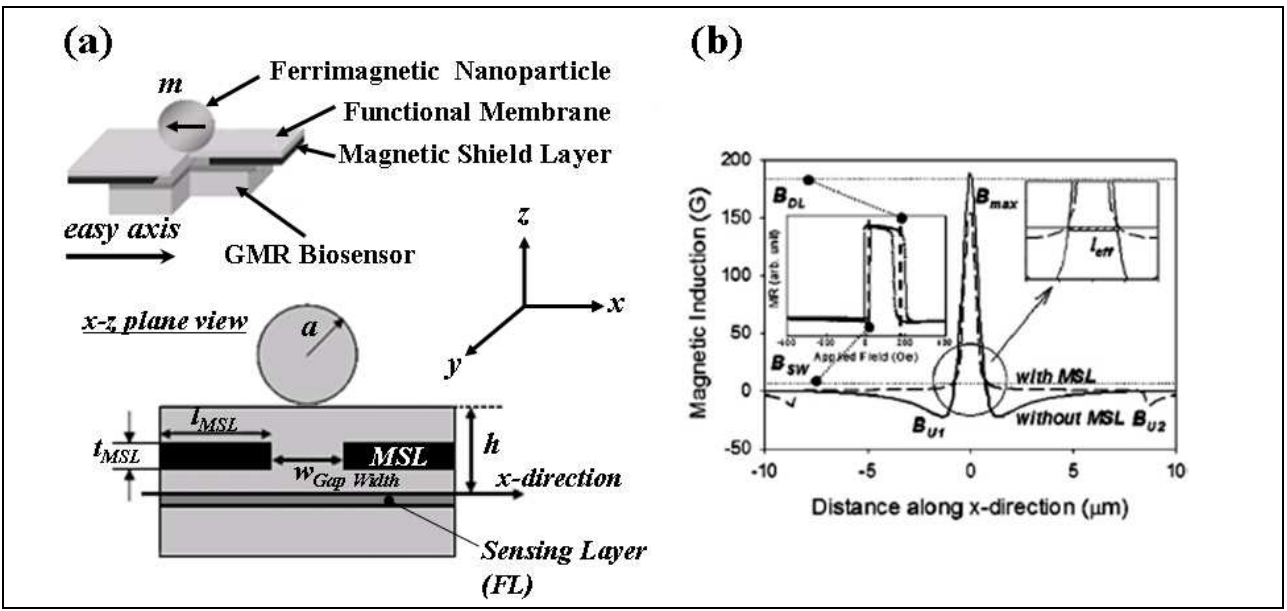

Fig. 10. (a) A schematic diagram of an in-vitro GMR biosensor with a specially designed MSL, and (b) the distribution of $x$-component of stray field produced by an immobilized single $\mathrm{CoFe}_{2} \mathrm{O}_{4}$ FNSA, with (solid line) and without (dashed line) MSL

For the numerical calculation based on equation (14), a radius of $250 \mathrm{~nm}$ size $\mathrm{CoFe}_{2} \mathrm{O}_{4}$ FNSA, an $176 \mathrm{G}$ of exchange bias field, and a $0.48 \mu \mathrm{m}$ of $h$ are considered. As can be seen in Fig. 10-(a), the calcualted $h$ value includes FL/passivation layer $(40 \mathrm{~nm}) / \mathrm{MSL}(0 \sim 300$ $\mathrm{nm}) / \mathrm{Al}_{2} \mathrm{O}_{3}$ functional membrane $(140 \mathrm{~nm}$; including a length of biological entities such as ten sequence of DNA: $34 \mathrm{~nm}$ ). The $\mathrm{Al}_{2} \mathrm{O}_{3}$ functional membrane layer in this sensor structure is used for both maintaing the $h$ depending on the variation of MSL thickness and immobilizing the FNSA using a membrane probe. The high magnetic permeability of materials such as supermalloy and permally are considered as a MSL in this structure. Figure 10-(b) shows a longitudinal field component ( $x$ direction) of stray field intesity on the FL surface without (solid line) and with (dashed line) MSL. The distribution of magentic field intensity shown in Fig. 10-(b) is calcualted as a function of distance from the center point of FNSA to the edge of GMR biosensor along the $x$ direction. In addition, $B_{\max }, B_{U 1}$, $\mathrm{B}_{\mathrm{U} 2}$, and $l_{\text {eff }}$ are denoted as a maximum magentic field intensity, an undershoot field, an outer undershoot field, and an effective detectable length, which is the region enabled to be reversed by the stray field, respectively. 


\subsection{Effects of magnetic shield layer (MSL) on the sensing performance of in-vitro GMR biosensor for SMD}

The MSL has a basic geometry of $300 \mathrm{~nm}$ thickness, a permeability of supermalloy $(5.2 \times$ $\left.10^{5}\right), 1.2 \mu \mathrm{m}$ gap width, and $8 \mu \mathrm{m}$ length. To explore the effects of MSL on the sensing performance, the physical parameters of the MSL including its thickness, its gap width, and its permeability are changed from the basic structure. Figure 11 shows the dependence of MSL thickness on the sensing performance compared in terms of the physical sensing parameters such as $B_{\mathrm{U} 1}, B_{\max }, l_{\mathrm{eff}}$, and $B_{\mathrm{U} 2}$. As can be seen in Fig. 11-(a), the undesirable "undershoot field region", $B_{\mathrm{U} 1}$, is dramatically reduced when the MSL has a $1 \mathrm{~nm}$ of thickness. By further increasing its thickness, $B_{\mathrm{U} 1}$ is sharply decreased and then it is completely removed above $t_{\mathrm{MSL}}=100 \mathrm{~nm}$. This indicates that the MSL needs to have a critical thickness to build up a close magnetic flux between the FNSA and the MSLs allowing for a distinct magnetic shielding effect. Figure 11-(b) shows the dependence of MSL thickness on the change of $B_{\max }$. When the MSL has a $10 \mathrm{~nm}$ of thickness and above, the $B_{\max }$ is decreased down to $158 \mathrm{G}$ and then it saturates at $153 \mathrm{G}$ by further increasing the MSL thickness above $100 \mathrm{~nm}$. This numerical result along with the increase of $l_{\text {eff }}$ shown in Fig. 11-(c) indicates that the effects of MSL on the improvement of sensing performance of the invitro GMR biosensor are quite prominent. The reduction of $B_{\max }$ from $188 \mathrm{G}$ (above $B_{\mathrm{DL}}$ ) to $155 \mathrm{G}$ (below the exchange bias field degradation point) and the increase of $l_{c}$ of the sensing area due to the MSL shielding effects allow the dramatic increase of "effective sensing area" on the FL surface that leads to the increase of sensing output signal of the patterned GMR biosensor. However, as can be seen in Fig. 11-(d), the magnetic dipole field induced in the MSL due to its high magnetic moment generates another undesirable small "undershoot field", $B_{\mathrm{U} 2}$ at the vicinity of the MSL edges. Even though its numerical value is small below

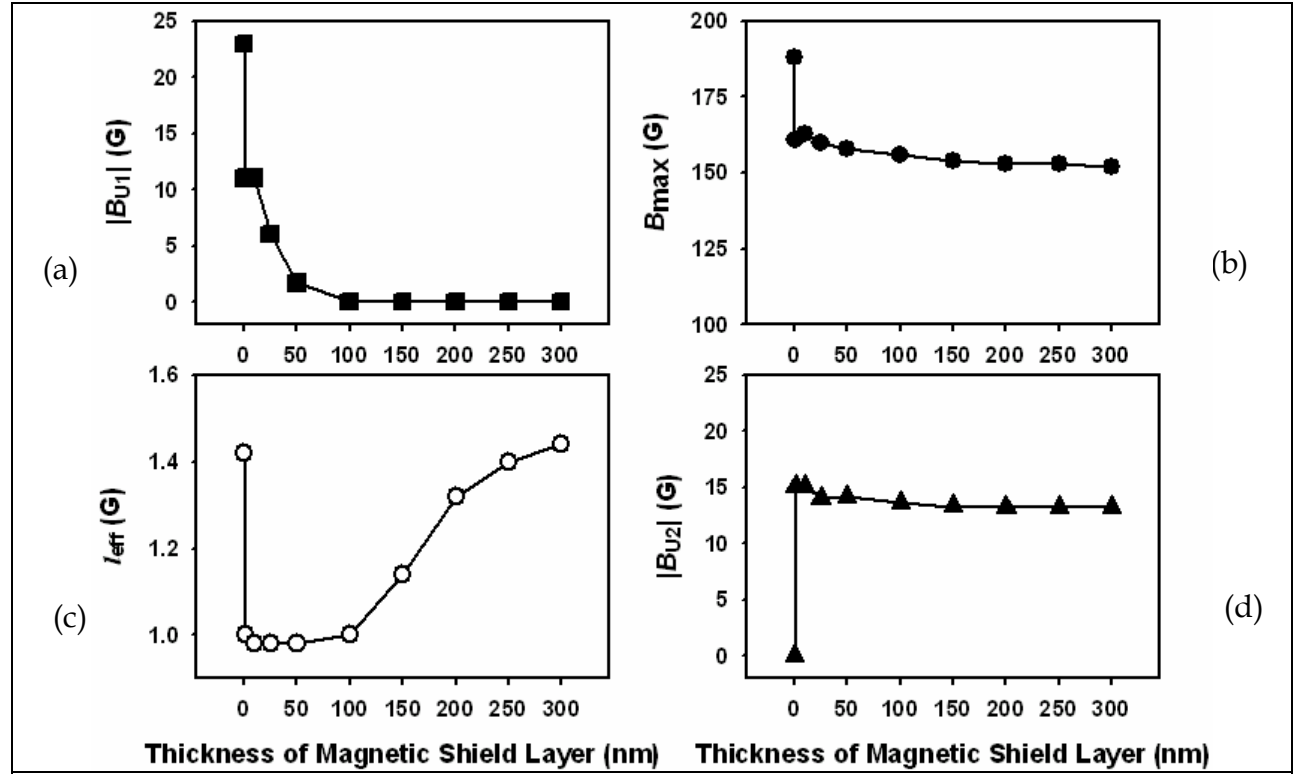

Fig. 11. Effects of MSL thickness on the sensing performance of an in-vitro GMR biosensor with an immobilized $250 \mathrm{~nm}$ size $\mathrm{CoFe}_{2} \mathrm{O}_{4}$ FNSA. (a) $B_{\mathrm{U} 1}$, (b) $B_{\max }$, (c) $l_{\text {eff, }}$ and (d) $B_{\mathrm{U} 2}$ 
12 G, it should be carefully controlled when the MSL is used for especially multi-array sensor architecture. The effects of MSL permeability on the sensing performance is not discussed in details in this chapter as all the physical sensing parameters have the same dependence on the permeability of MSL. However, the numerical calculation results obtained from the GMR biosensor with geometry of a $300 \mathrm{~nm}$ MSL thickness, a $1.2 \mu \mathrm{m}$ of gap width, and a $8 \mu \mathrm{m}$ of MSL length demonstrates that the MSL with magnetic permeability of at least 100 shows obvious shielding effects. Figure 12 shows the dependence of MSL gap width, which is changed from 0.2 to $2.0 \mu \mathrm{m}$, on the sensing performance of an in-vitro GMR biosensor with an immobilized $\mathrm{CoFe}_{2} \mathrm{O}_{4}$ FNSA. As shown in Fig. 12-(a), the $B_{\max }$ is dramatically increased from $150 \mathrm{G}$ to $260 \mathrm{G}$, which is beyond the sensing limit, $\mathrm{B}_{\mathrm{DL}}$, by decreasing the gap width from 0.8 to $0.2 \mu \mathrm{m}$. The dramatic increase of magnetic field intensity at the gap width below $0.8 \mu \mathrm{m}$ is mainly thought to be attributed to the increase of fringe field produced from the gap between two MSLs (This is quite similar to the "fringe field" produced by the head gap from the writer in magnetic recording technology) (Bertram, 1994). The high permeability of two MSLs, which are closely faced each others with small gap, can produce a strong "deep gap bubble field" due to a high magnetic flux density. This leads to increasing the "fringe field" on the surface of MSL gap that would be diverged into the FL surface resulting in the increase of magnetic field intensity on the FL (sensing layer) surface.

As shown in Figs. 12-(b) and 12-(c), the $B_{\mathrm{U} 1}$ and the $l_{\text {eff }}$ are also strongly influenced by the MSL gap width. The $B_{\mathrm{U} 1}$ is obviously re-developed when the MSL gap width is increased above $1.4 \mu \mathrm{m}$. Moreover, $l_{\text {eff }}$ is sharply decreased when the MSL gap width is increased above $1.2 \mu \mathrm{m}$. The serious degradation of sensing performance relevant to the dramatic

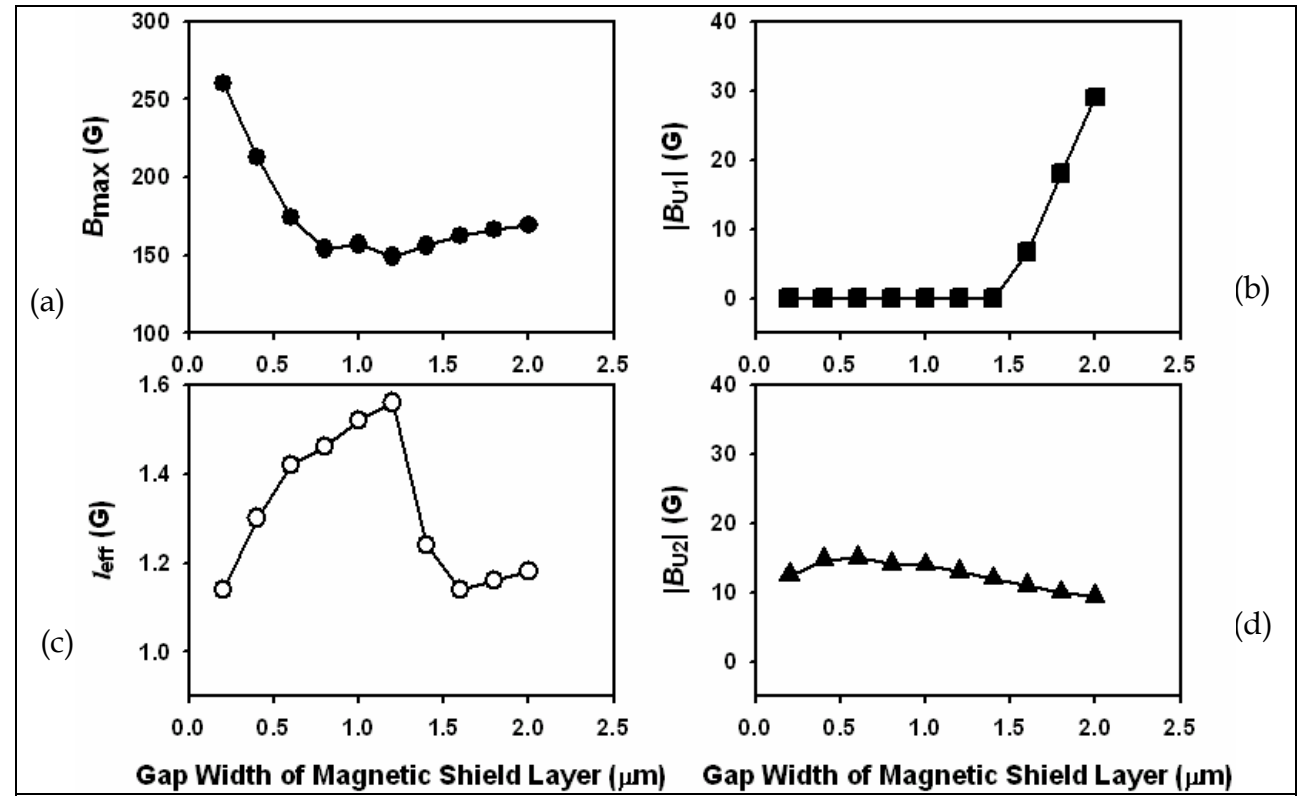

Fig. 12. Effects of MSL gap width on the sensing performance of an in-vitro GMR biosensor with an immobilized $250 \mathrm{~nm}$ size $\mathrm{CoFe}_{2} \mathrm{O}_{4}$ FNSA. (a) $B_{\mathrm{U} 1}$, (b) $B_{\max }$ (c) $l_{\text {eff, }}$ and (d) $B_{\mathrm{U} 2}$ 
reduction of "effective sensing area" on the sensor surface due to both the re-development of "undershoot field region" and the reduction of critical length, $l_{c}$, are mainly attributed to the reduction of shield gap flux density, which is inversely proportional to the MSL gap width. However, considering that the $l_{c}(\sim 1.56 \mu \mathrm{m})$, which is numerically determined based on the same geometry and configuration of in-vitro GMR biosensor system, is found to be much larger than that without MSL $(\sim 1.12 \mu \mathrm{m})$, it can be readily understood that shielding effects of MSL on the improvement of sensing performance is quite significant.

In summary, it is numerically demonstrated that the MSL is effective to improve the output sensing performance of an in-vitro GMR biosensor with an immobilized FNSA, because it can successfully remove an undesirable "undershoot field region" and enhance the "effective sensing area" due to the increase of $l_{c}$. This indicates that an in-vitro GMR biosensor with a specially designed MSL structure can be considered as a promising sensor structure for SMD due to its achievable high sensing signal and stability.

\section{Conclusion}

The physical sensing characteristics of an in-vitro GMR biosensor with an immobilized single FNSA have been introduced and discussed to explore its feasibility to a single molecular based disease diagnostic biosensor system. According to the theoretically and experimentally analyzed results, the in-vitro GMR biosensor with a FNSA was revealed to be more suitable for SMD than that with a SPNSA due to its higher relative MR, less interaction factor dependence, and practically allowable sensor size. In addition, the analytical models developed in this chapter allowed to readily predicting the optimized sensor geometry of an in-vitro GMR biosensor with a FNSA prior to fabrication if the physical parameters of the FNSA are provided. In particular, the in-vitro GMR biosensor with a specially designed magnetic shield layer (MSL) was demonstrated to be able to effectively control the undesirable "undershoot sensing region" as well as the "undetectable sensing area" on the FL surface. These promising sensing characteristics improved by the newly designed sensor structure allow for achieving both maximized output sensing signal and higher sensor stability that lead to accelerating the more practical applications to the SMD based diagnostic biosensor system.

\section{References}

Baselt D. R.; Lee G. U.; Natesan M.; Metzger S. W.; Sheehan P. E. \& Colton R. J., (1998). A biosensor based on magnetoresistive technology. Biosensors and Bioelectronics, Vol. 13, pp. 731-739, ISSN 0956-5663

Besse P.; Boero G.; Demierre M.; Pott V. \& Popovic R., (2002). Detection of a single magnetic microbead using a miniaturized silicon Hall sensor. Applied Physics Letters, Vol. 80, No. 22, pp. 4199-4201, ISSN 0003-6951

Bertram, N. (1994). Theory of magnetic recording, Cambridge, ISBN 0-521-44512-4, New York

Girgis E.; Schelten J.; Shi J., Tehrani S. \& Goronicin H., (2000). Switching characteristics and magnetization vortices of thin-film cobalt in nanometer-scale patterned arrays. Applied Physics Letters, Vol. 76, No. 25, pp. 3780-3782, ISSN 0003-6951

Graham D. L.; Ferreira H.; Bernardo J.; Freitas P. P. \& Cabral J. M. S., (2000). Single magnetic microsphere placement and detection on-chip using current line designs with 
integrated spin valve sensors: Biotechnological applications. Journal of Applied Physics, Vol. 91, No. 10, pp. 7786-7788, ISSN 0021-8979

Graham D. L.; Ferreira H. A.; Freitas P.P. \& Cabral J. M. S., (2003). High sensitivity detection of molecular recognition using magnetically labeled biomolecules and magnetoresistive sensors. Biosensors and Bioelectronics, Vol. 18, pp. 483-488, ISSN 0956-5663

Graham D. L.; Ferreira H. A. \& Freitas P.P., (2004). Magnetoresistive-based biosensors and biochips. TRENDS in biotechnology, Vol. 22, No. 9, pp. 455-462, ISSN 0167-7799

Hirota E.; Sakakima H. \& Inomata K., (2002). Giant Magnetoresistance Device, Springer, ISBN 3-540-41819-9, Berlin

Lagae L.; Wirix-Speethens R.; Das J.; Graham D. L.; Ferreira H.; Freitas P. P.; Borghs G. \& Boeck J. De., (2002). On-chip manipulation and magnetization assessment of magnetic bead ensembles by integrated spin-valve sensors. Journal of Applied Physics, Vol. 91, No. 10, pp. 7445-7447, ISSN 0021-8979

Latham A. H.; Tarpara A. N. \& Williams M. E., (2007). Magnetic field switching of nanoparticles between orthpgonal microfluidic channels, Analytical Chemistry, Vol. 79, pp. 5746-5752, ISSN 10.1021

Lee S.; Bae S.; Takemura Y.; Shim I. B.; Kim T. M.; Kim J.; Lee H. J.; Zurn S. \& Kim C., (2007). Self-heating characteristics of cobalt ferrite nanoparticles for hypertheria application. Journal of Magnetism and Magnetic Materials, Vol. 310, pp. 2868-2870, ISSN 0304-8853

Li G. \& Wang S. X., (2003). Analytical and micromagnetic modeling for detection og a single magnetic microbead or nanobead by spin valve sensors. IEEE Transaction on Magnetics, Vol. 39, No. 5, pp. 3313-3315, ISSN 10.1109

Megens M. \& Prins M., (2005). Magnetic biochips: a new option for sensitive diagnostics. Journal of Magnetism and Magnetic Materials, Vol. 293, pp. 702-708, ISSN 0304-8853

Miller M. M.; Sheehan P. E.; Edelstein R. L.; Tamanha C. R.; Zhong L.; Bounnak S.; Whiteman L. J. \& Colton R. J., (2001). A DNA array sensor utilizing microbeads and magnetoelectronic detection. Journal of Magnetism and Magnetic Materials, Vol. 225, pp. 138-144, ISSN 0304-8853

Ramadan Q.; Samper V.; Poenar D. \& Yu C., (2006). Magnetic-based microfluidic platform for biomolecular separation, Biomedical Microdevices, Vol. 8, No. 4, pp. 151-158, ISSN 10.1007

Rife J. C.; Miller M. M.; Sheehan P. E.; Tamanaha C. R.; Tondra M. \& Whitman L. J., (2003). Design and performance of GMR sensors for the detection of magnetic microbeads in biosensors. Sensors and Actuators A, Vol. 107, pp. 209-218, ISSN 0924-4247

Schepper W.; Schotter J.; Bruckl H. \& Reiss G., (2004). Analyzing a magnetic molecule detection system - computer simulation. Journal of Biotechnology, Vol. 112, pp. 35-46, ISSN 0168-1656

Schepper W.; Schotter J.; Bruckl H. \& Reiss G., (2006). A magnetic molecule detection system-A comparison of different setups by computer simulation. Physica B, Vol. 372, pp. 337-340, ISSN 0921-4526

Shen W.; Liu X.; Mazumdar D. \& Xiao G., (2005). In-situ detection of singl micron-sized magnetic beads using magnetic tunnel junction sensors. Applied Physics Letters, Vol. 86, pp. 253901-253903, 0003-6951 
Stoner E. C. \& Wöhlfarth, (1948). A mechanism of magnetic hysteresis in heterogeneous alloys. Philosophical Transactions of the Royal Society A, Vol. 240, pp. 599-642, ISSN 1471-2961

Tondra M.; Porter M. \& Lipert R. J., (2000). Model for detection of immobilized superparamagnetic nanosphere assay labels using giant magnetoresistive sensors. Journal of Vacuum Science and Technology A, Vol. 18, No. 4, pp. 1125-1129, ISSN 07342101

Wang S. X.; Bae S. Y.; Li G.; Sun S.; White R. L., Kemp J. T. \& Webb C. D., (2005). Towards a magnetic microarray for sensitive diagnostics. Journal of Magnetism and Magnetic Materials, Vol. 293, pp. 731-736, ISSN 0304-8853

William E. M., (2001). Design and analysis of magnetoresistive recording heads, John Wiley \& Sons, ISBN 0-471-36358-8, New York

Wirix-Speetjens R.; Fyen W.; Boeck J. D. \& Borghs G., (2006). Single magentic particle detection: Experimental verification of simulated behavior. Journal of Applied Physics, Vol. 99, No. 103903, pp. 1-4, ISSN 0021-8979 


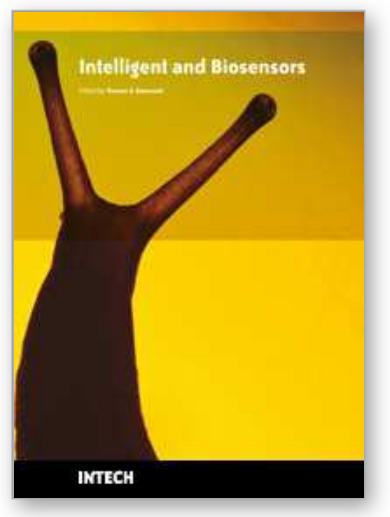

\author{
Intelligent and Biosensors \\ Edited by Vernon S. Somerset
}

ISBN 978-953-7619-58-9

Hard cover, 386 pages

Publisher InTech

Published online 01, January, 2010

Published in print edition January, 2010

The use of intelligent sensors have revolutionized the way in which we gather data from the world around us, how we extract useful information from that data, and the manner in which we use the newly obtained information for various operations and decision making. This book is an attempt to highlight the current research in the field of Intelligent and Biosensors, thereby describing state-of-the-art techniques in the field and emerging new technologies, also showcasing some examples and applications.

\title{
How to reference
}

In order to correctly reference this scholarly work, feel free to copy and paste the following:

Seongtae Bae (2010). In-Vitro Magnetoresistive Biosensors for Single Molecular Based Disease Diagnostics: Optimization of Sensor Geometry and Structure, Intelligent and Biosensors, Vernon S. Somerset (Ed.), ISBN: 978-953-7619-58-9, InTech, Available from: http://www.intechopen.com/books/intelligent-and-biosensors/invitro-magnetoresistive-biosensors-for-single-molecular-based-disease-diagnostics-optimization-of-

\section{INTECH}

open science | open minds

\section{InTech Europe}

University Campus STeP Ri

Slavka Krautzeka 83/A

51000 Rijeka, Croatia

Phone: +385 (51) 770447

Fax: +385 (51) 686166

www.intechopen.com

\section{InTech China}

Unit 405, Office Block, Hotel Equatorial Shanghai

No.65, Yan An Road (West), Shanghai, 200040, China

中国上海市延安西路65号上海国际贵都大饭店办公楼 405 单元

Phone: +86-21-62489820

Fax: $+86-21-62489821$ 
(C) 2010 The Author(s). Licensee IntechOpen. This chapter is distributed under the terms of the Creative Commons Attribution-NonCommercialShareAlike-3.0 License, which permits use, distribution and reproduction for non-commercial purposes, provided the original is properly cited and derivative works building on this content are distributed under the same license. 